\title{
First-Principles Calculation on Electronic Properties of Bismuth-halide Inorganic Perovskites for Solar Cell
}

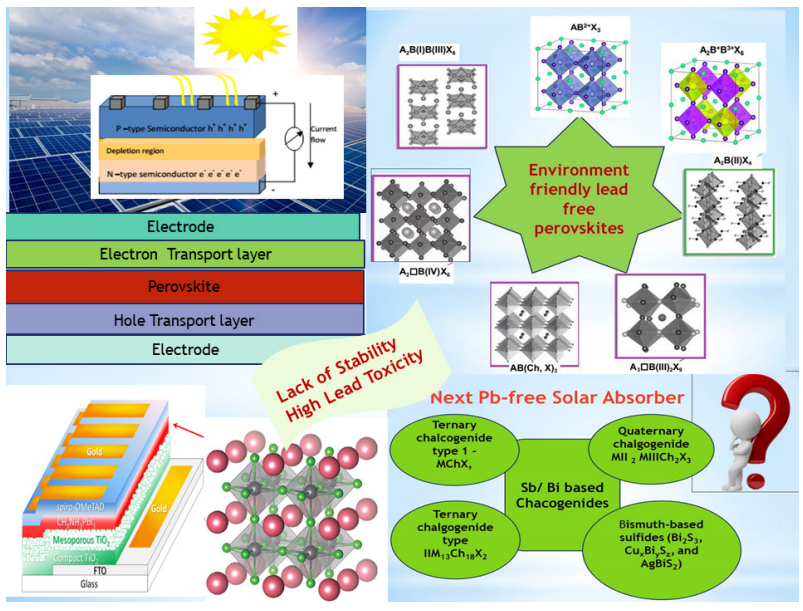

Solar energy is a commonly used alternate source of energy and it can be utilized based on the principle of the photovoltaic effect. The photovoltaic effect converts sun energy into electrical energy using photovoltaic devices (solar cells). A solar cell device should have high efficiency and a long lifetime to be commercially beneficial. Presently, silicon and thin-film solar cells are widely employed. The crystalline solar cells are more efficient but they are also expensive. Thin-film solar cells are formed by placing one or more thin layers of photovoltaic materials on different substrates. Although these cells have a lower cost, they are also less efficient compared to Si-based solar cells. Organic-inorganic hybrid lead halide perovskite solar cells are one of the most promising low-cost power conversion efficiency technologies that could exceed the $26 \%$ threshold. However, the lack of environmental stability and of high lead toxicity are the main bottlenecks that impede the future industrialization and commercialization hybrid lead halide perovskite. Hence It is important to achieve high power conversion efficiency while also maintaining stability and non-toxicity in the development of new lead-free perovskite materials.
Bi-based halide perovskites are an interesting class of material because of theirrich structural diversity and semiconducting properties. The ability of Bismuth to exist in a wide range of coordination geometries (from cluster to 1D, 2D, or even 3D) makes it particularly useful for designing novel compounds for optoelectronic applications. Studies on the coordination chemistry of Bismuth halides over several decades conclude that the network of metal anionic motifs can be easily manipulated by varying the size and geometry of cations, reaction environments, and crystallization conditions. Based on recent success with $\mathrm{Pb}$ based halide perovskites, similar attempts have been made to synthesize Bi-based perovskites which crystallize into $\left[\mathrm{Bi}_{2} \mathrm{l}_{9}\right]^{3-}$ family of compounds. Herein, we examined the electronic properties of $\mathrm{Cs}_{3} \mathrm{Bi}_{2} \mathrm{I}_{9}$ as a prototype $\mathrm{Bi}$-based iodide perovskite for photovoltaic applications.

The electronic structure calculations are performed through the Vienna ab initio simulation package (VASP) with the standard frozen-core projected augmented-wave method (PAW). The atomic positions are relaxed till the force/atom is less than $0.05 \mathrm{eV} / \AA$. All structural relaxations were performed with Gaussian smearing of $0.05 \mathrm{eV}$. Cs, Bi, and $I$ atoms are described by $5 s^{2} 5 p^{6} 6 s^{1}, 5 d^{10} 6 s^{2} 6 p^{3}$, $5 s^{2} 5 p^{5}$ valence electrons respectively. The simulations of the band structures were carried out in two steps. Initially, the unit cell was relaxed by a self-consistent run. Finally, a non-self-consistent run was performed using earlier charge density and the energy states were calculated along high symmetry direction on discrete $\mathrm{K}$ mesh. The high symmetry path is shown in Figure 1 from Brillouin zone center $\Gamma \mathrm{m}$, with the coordinates $(0,0,0)$ to $M$ $(0.5,0,0), K(0.333,0.333,0), \Gamma(0,0,0), A(0,0,0.5), L$ $(0.5,0,0.5)$, and $H(0.333,0.333,0.5)$. All schemat- 


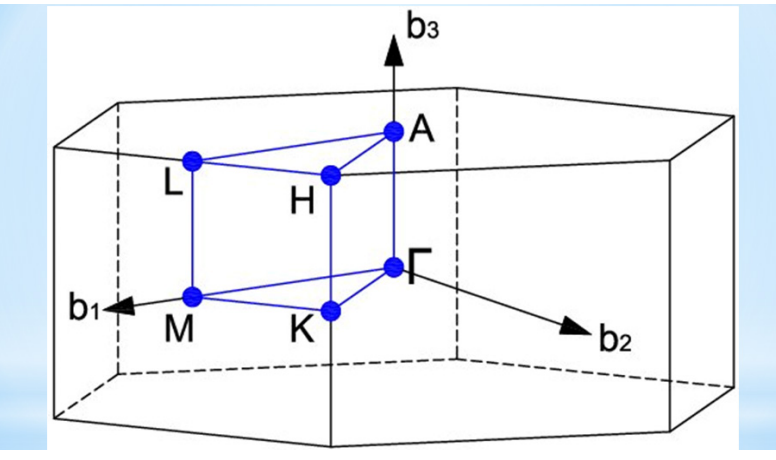

Figure 1: High symmetry path in the hexagonal Brillouin zone

ic representations of the crystal structures were generated using the VESTA program [1].

\section{Crystal Structure}

$\mathrm{Cs}_{3} \mathrm{Bi}_{2} \mathrm{I}_{9}$ exhibits hexagonal crystal symmetry at room temperature with the space group $\mathrm{P}_{3} / \mathrm{mmc}$ and undergoes a ferroelastic phase transition to a monoclinic structure at $220 \mathrm{~K}$ [2]. The hexagonal structure can be considered as a distorted and defect modulated face-sharing perovskite structure in which every third layer of octahedral Bi sites is depleted for charge compensation. Consequently, two neighbouring $\left[\mathrm{Bil}_{6}\right]^{3-}$ octahedrons form a face-sharing $\left[\mathrm{Bi}_{2} l_{9}\right]^{3-}$ bi - octahedra which are separated by $\mathrm{Cs}+$ cations, eventually forming a $\mathrm{OD}$ crystal structure.

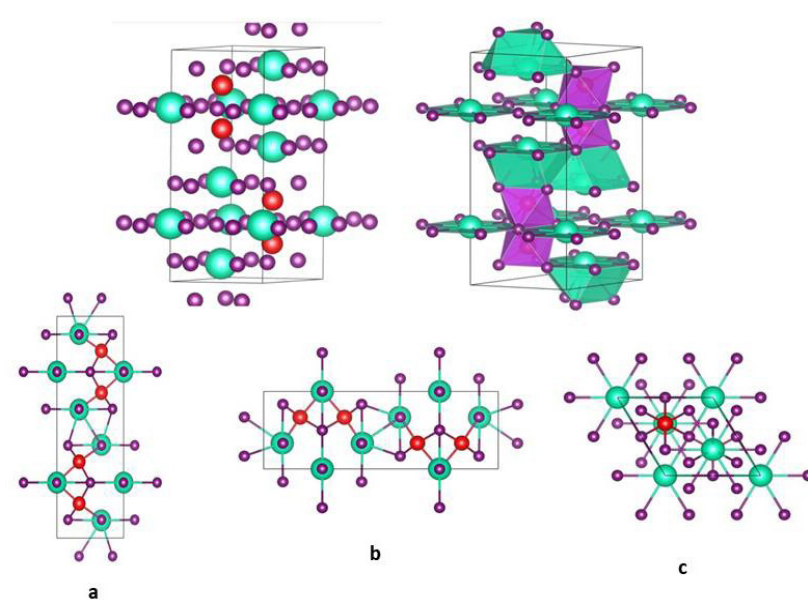

Figure 2: Schematic crystal structure of $\mathrm{Cs}_{3} \mathrm{Bi}_{2} \mathrm{I}_{9}$ (red, green, and violet colours represent $\mathrm{Bi}, \mathrm{Cs}$, and I atoms) as seen along (a) a-axis, (b) b-axis, and (c) c-axis

The Density of state and Band Structure

of $\mathrm{Cs}_{3} \mathrm{Bi}_{2} \mathrm{l}_{9}$

The VBM is arbitrarily set at $0 \mathrm{eV}$ and is located at the $\mathrm{M}$ point. According to our calculations, the electronic bandgap is indirect between $\Gamma$ and $M$

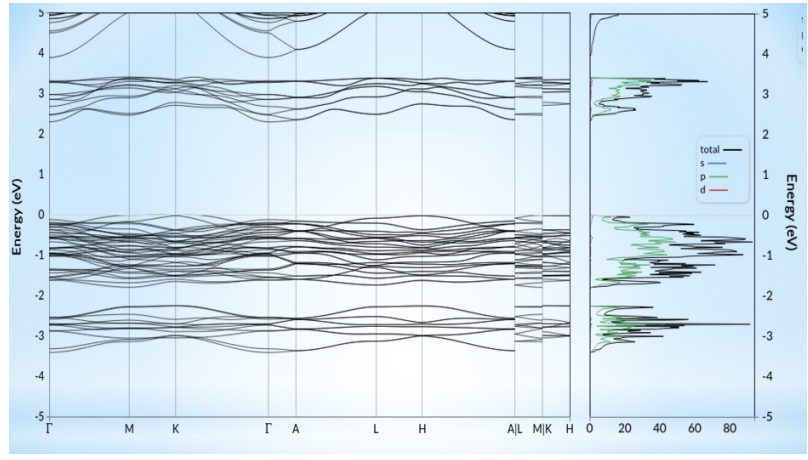

Figure 3: Density of state and band structure of $\mathrm{Cs}_{3} \mathrm{Bi}_{2} \mathrm{l}_{9}$

point. The calculated bandgap value is found to be $2.34 \mathrm{eV}$.

The orbital contribution of the Cs+ ion can easily be recognized by the vertical line in the density of state; this indicates that the electronic states of Cs are fully localized in space, without substantial interaction with inorganic bioctahedra. The valence band of $\mathrm{Cs}_{3} \mathrm{Bi}_{2} \mathrm{l}_{9}$ consists of the predominant contributions from $p$ orbital of I and a little contribution from Bi(s) orbitals. The CBM is composed of nearly equal contributions from $\mathrm{I}(\mathrm{p})$ and $\mathrm{Bi}(\mathrm{p})$ orbitals. From these results, a paramount feature of this compound emerges: $\mathrm{Cs}$ and inorganic $\left[\left.\mathrm{Bi}_{2}\right|_{9}\right]^{3-}$ cages are decoupled from the electronic viewpoint and therefore, $\mathrm{Cs}$ do not interfere with the active region of the perovskite. Its only role, regarding the electronic properties, is to donate one electron to the surrounding environment.

\section{References}

[1].V. F. Machulin, F. V. Motsnyi, O. M. Smolanka, G. S. Svechnikov, andE. Y. Peresh, "Effect of temperature variation on shift and broadening ofthe exciton band in Cs3Bi219 layered crystals," Low Temp. Phys., vol. 30,pp. 964-967, 2004.

[2].K. Momma and F. Izumi, "VESTA 3 for three-dimensional visualizationof crystal, volumetric and morphology data," J. Appl. Crystallogr., vol.44, pp. 1272-1276, 2011.

[3].W. Bo, N. H. Tiep, K. Zhiliang, H. Guifang, G. David, M. Nripan, et al.,"Discerning the Surface and Bulk Recombination Kinetics of Organic-Inorganic Halide Perovskite Single Crystals," Adv. Energy Mater., vol. 6,p. 1600551, 2016

Article by

Galhenage A. Sewvandi \& J.T.S.T.Jayawardane Department of Materials Science and Engineering, Faculty of Engineering, University of Moratuwa, Sri Lanka 